\title{
A new derived species group of Aleiodes parasitoid wasps (Hymenoptera, Braconidae, Rogadinae) from Asia with descriptions of three new species
}

\author{
Buntika Areekul Butcher ${ }^{1, \dagger}$, M. Alex Smith ${ }^{2, \ddagger}$, Donald L.J. Quicke ${ }^{3,4, \S}$ \\ I Department of Biology, Faculty of Science, Chulalongkorn University, BKK 10330, Thailand 2 Department \\ of Integrative Biology, Biodiversity Institute of Ontario, University of Guelph, Guelph, Ontario N1G 2W1, \\ Canada 3 Department of Biology, Imperial College London, Silwood Park Campus, Ascot, Berks SL5 7PY, UK \\ 4 Department of Entomology, Natural History Museum, London SW7 5BD, UK \\ † urn:lsid:zoobank.org:author:3AC486D1-9045-44FB-9FE4-FF71A0C2909C \\ † urn:lsid:zoobank.org:author:E46EE6EB-E096-4FCD-BF5A-F91D4A8294EE \\ § urn:lsid:zoobank.org:author:4FAA3C17-13D3-467B-AAA5-82B27FE783EF \\ Corresponding author: Donald L.J. Quicke (d.quicke@imperial.ac.uk)
}

Academic editor: Gavin Broad | Received 4 June 2011 | Accepted 26 August 2011 | Published 21 October 2011

urn:lsid:zoobank.org:pub:82E3228A-2959-4494-A033-CF4D8479C2E0

Citation: Butcher BA, Smith MA, Quicke DLJ (2011) A new derived species group of Aleiodes parasitoid wasps (Hymenoptera, Braconidae, Rogadinae) from Asia with descriptions of three new species. Journal of Hymenoptera Research 23: 35-42. doi: 10.3897/JHR.23.1663

\begin{abstract}
Three new species of Asian Aleiodes are described and illustrated: A. spurivena sp. n. from Vietnam, Thailand, Nepal and India; A. spurivenaduplus sp. n. from Vietnam; A. spurivenatriplus from Western Malaysia. The first two of these are differentiated based on the bar-coding, 5' region of the cytochrome oxidase 1 gene. All three are morphologically extremely uniform and can be recognised from other Aleiodes species by the presence of a reclivous spur arising from fore wing vein 1-1A.
\end{abstract}

\section{Keywords}

Aleiodini, Oriental, cryptic species, colour variation 


\section{Introduction}

During revision of the species of the rogadine braconid wasp genus Aleiodes Wesmael formerly classified in the genus Hemigyroneuron (Areekul Butcher \& Quicke, 2011), we encountered a number of superficially similar specimens from India, Nepal, Thailand, Vietnam and peninsular Malaysia, that represent an easily recognised, previously undescribed species group. The specimens are large and brightly coloured yellow and black. Specimens collectively show marked differences in colour pattern, but are morphologically very difficult to distinguish. We have obtained DNA sequence data for four of the specimens (all from Vietnam) that indicate the presence of two species differing only slightly in wing venation. Based on morphology alone, one of these appears to be widespread in mainland Asia. Here we describe this new species group to include these two species along with a third from Malaysia that displays slightly more derived wing venation and darker coloration. Based on limited collecting evidence, we suspect much of the colour variation is intraspecific.

Terminology follows van Achterberg (1996) repeated in Chen and He (1997). Sculptural terms follow Harris (1979). The following abbreviation is used: BMNH, Natural History Museum, London; RMNH, Naturalis Museum, Leiden, Netherlands.

\section{Systematics}

\section{Diagnosis of Aleiodes spurivena sp. n. species group}

Occipital carina complete. Propodeum with distinct medio-lateral angulations and with more or less complete mid-longitudinal carina. Fore wing vein 1-A with a posterior short to long spur; distal 0.7 or more of subbasal cell glabrous (except ventrally narrowly along anterior margin) and distally swollen but lacking sclerome; junction of veins $\mathrm{M}+\mathrm{CU}, 1-\mathrm{CU} 1$ and 1-M moderately to very strongly swollen; subdiscal cell evenly setose; 2 nd submarginal cell short and trapezoidal. Hind wing with vein $r$ present as an oblique sclerotized line running at least a third of width of marginal cell. Apex of hind tibia with well-developed comb of specialised flattened setae on medial and ventral faces. Claws with strong pecten of approximately 12 teeth nearly reaching base of claw. Male without median tergal gland pore on tergites 4-6.

\section{Key to the species of the $A$. spurivena sp. n. group}

1 Forewing veins $\mathrm{M}+\mathrm{CU}$ and 1-CU1 strongly swollen at junction with 1-M, vein $\mathrm{M}+\mathrm{CU}$ narrowed and distinctly sinuate before the swelling (Fig. 1a); 
hind femur beyond trochantellus and hind tibia entirely black

A. spurivenatriplus sp. $\mathrm{n}$.

- $\quad$ Forewing veins $\mathrm{M}+\mathrm{CU}$ and 1-CU1 weakly swollen at junction with 1-M, vein $\mathrm{M}+\mathrm{CU}$ not distinctly sinuate before the swelling (Figs 1b,c); hind femur black with apex yellow, hind tibia yellow on at least basal third (Fig. 2) ......2 2 Fore wing vein 1-CU1 (measured between middles of its swollen junctions) less than $0.6 \times$ length of 2-CU1 (Fig. 1b); spur on fore wing vein 1-A tubular and reaching close to posterior margin of wing (Fig. 1b)...A. spurivena sp. $\mathbf{n}$. - $\quad$ Fore wing vein 1-CU1 (measured between middles of its swollen junctions) $0.9 \times$ length of 2-CU1 (Fig. 1c); spur on fore wing vein 1-A short, not reaching beyond middle of anal cell (Fig. 1c)

A. spurivenaduplus sp. $\mathrm{n}$.

\section{Aleiodes spurivena Quicke \& Butcher, sp. $\mathrm{n}$.} urn:lsid:zoobank.org:act:05393EB9-AF1A-4D9A-B658-A30A05672DA3 http://species-id.net/wiki/Aleiodes_spurivena

Figs $1 b, 2,3$

Holotype female. "VIETNAM, Vihn Phu Prov., Thung Cuu, $30 \mathrm{~km} \mathrm{~S}$ of Than Son, 10-16.XI.1999, 20.59 N 105.08 E, 300m R. de Jong at light VN 99-8 RMNH 2000”, "BCLDQ0003" (RMNH).

Paratypes. VIETNAM: 2 females, Vihn Phu Province, Thung Cuu, (DNA vouchers BF00928, BF00932) (RMNH). THAILAND: 2 females, S. Thailand, $7 \mathrm{~km} \mathrm{~N}$ of Ranong, Ch 9 TV relay stn., 25-29.xi.1991, 350-500m, I.J. Kitching \& a. M. Cotton (BMNH); 1 female, Chiang Mai, Doi Suthap, 1440m, 1983 (BMNH). NEPAL: 1 female, Kathmandu, British Embassy, v.i.1984, 1350 m., M. G. Allen (BMNH). INDIA: 1 female, Assam, Nambour Forest Reserve, 29.xi.1997, Sinaer \& Murzin (private collection); 1 male, United Provinces, Naini Tal., c. 500ft., 15.iv.1934, "at light", J. A. Graham $(\mathrm{BMNH})$.

Coloration. Bright ochreous yellow with black pattern of variable extent. Minimally with flagellum, stemmaticum, metasomal tergite 4, hind coxa largely, hind femur medially, hind tibia apically, and hind tarsus black. In darker specimens black or dark brown marks occur on lateral lobes of mesoscutum, mesopleuron, propodeum antero-laterally, and metasomal tergites $2-4$. Wings pale yellow with yellow to brown yellow venation and with fore wing parastigma and apex of $\mathrm{C}+\mathrm{SC}+\mathrm{R}$ black.

Notes. The variation in coloration is quite large, however, we are inclined to think all the above specimens are conspecific because of the lack of any apparent morphological distinction, and because two of the most extreme extents of colour variation Fig. $3 \mathrm{~b}$ versus Fig $3 \mathrm{~d}$, were collected at the same site and on the same occasion. The specimen from India most closely resembles the holotype except that it has metasomal tergite 4 brown yellow instead of black. 

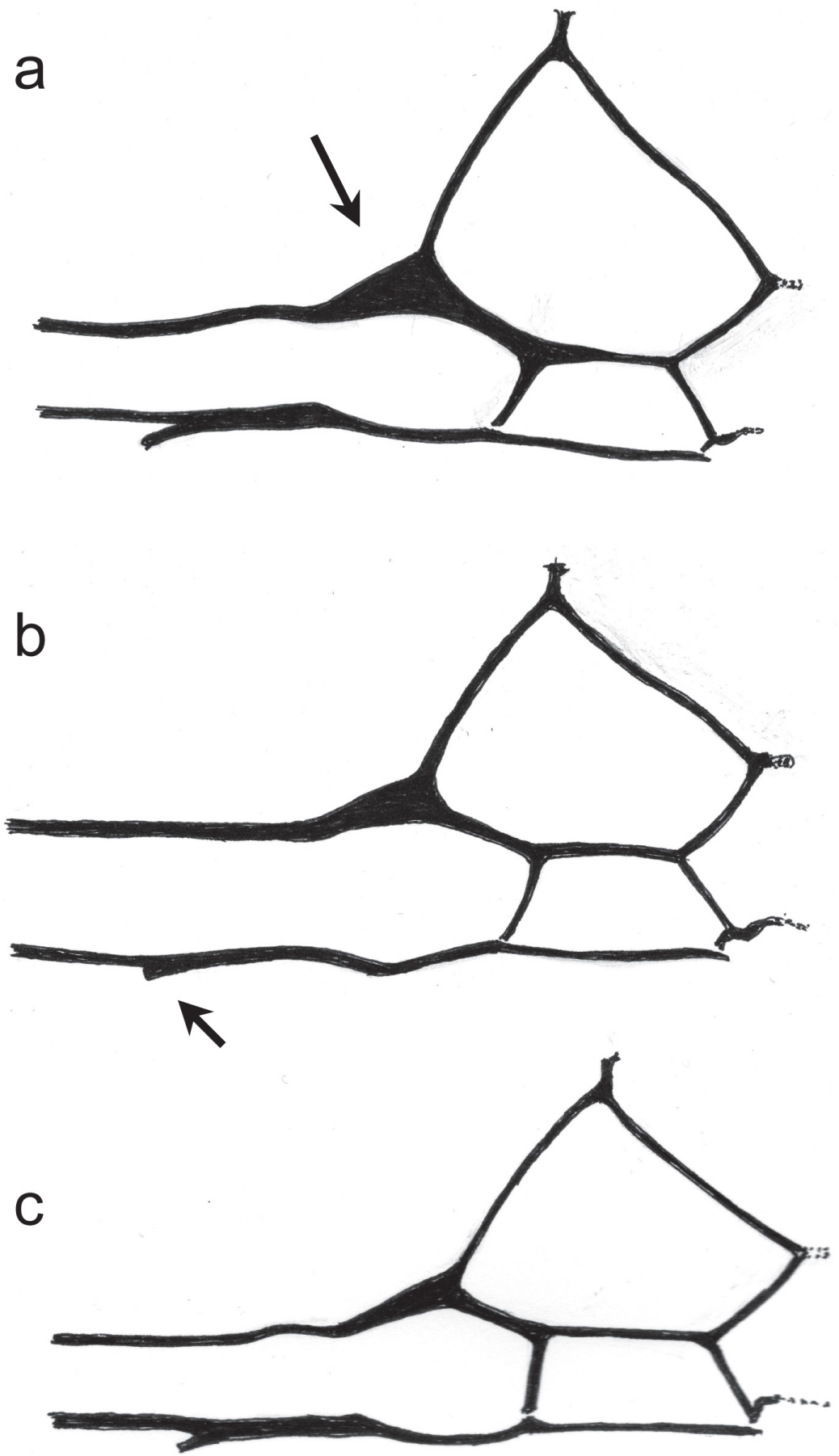

Figure I. Features of fore wing venation of $\mathbf{a}$ Aleiodes spurivenatriplus sp. n. b A. spurivena sp. n. $\mathbf{c} A$. spurivenaduplus sp. n. 


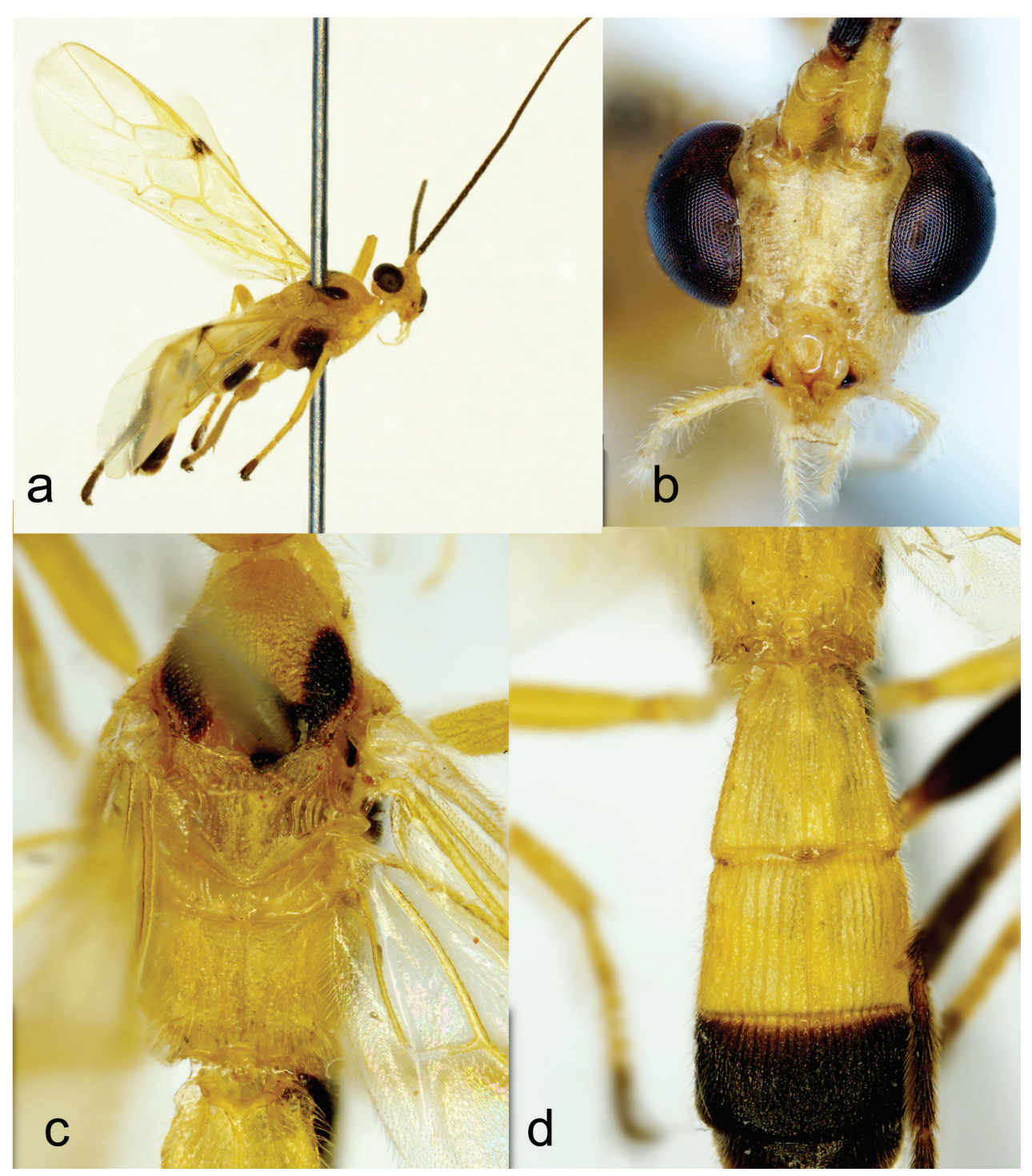

Figure 2. Cell^ ${ }^{\wedge}$ montage images of holotype of $A$. spurivena sp. $\mathrm{n}$. a habitus $\mathbf{b}$ face $\mathbf{c}$ mesosoma, dorsal view d metasoma.

\section{Aleiodes spurivenaduplus Quicke \& Butcher, sp. n.}

urn:Isid:zoobank.org:act:5B0D35F2-0ADB-41BA-A111-CACCC0800ABE http://species-id.net/wiki/Aleiodes_spurivenaduplus

Fig. 1c

Holotype female. "C. VIETNAM: Thua Thien Hué, Phong Dién N.R. nr base-camp, 50-100 m. 24.iii.2001. at light, C. v. Achterberg RMNH01”, "BCLDQ01315 [DNA voucher code]" (RMNH). 


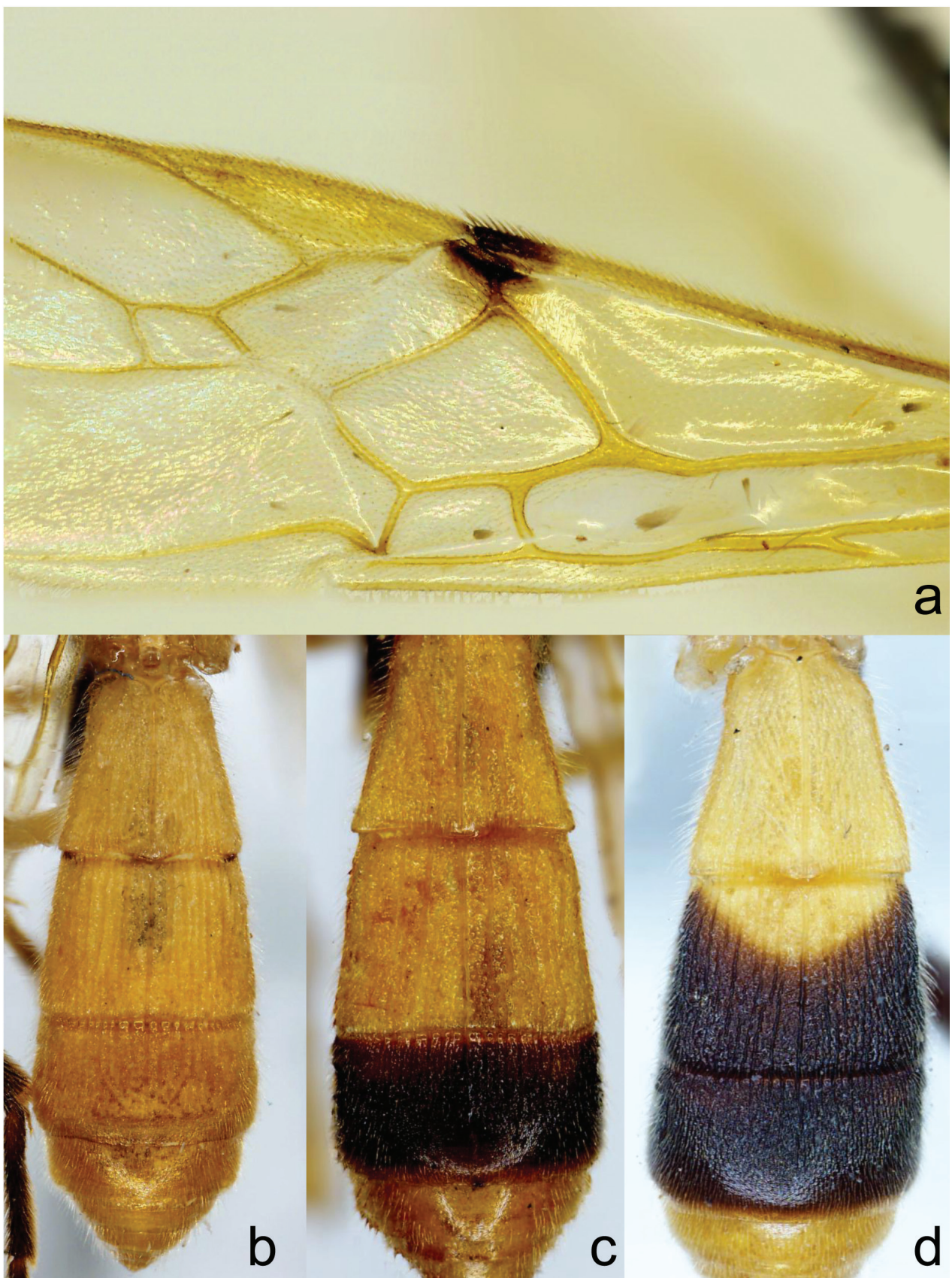

Figure 3. Cell^ $\mathrm{D}$ montage images of paratype $A$. spurivena sp. n. specimens a detail of fore wing venation $\mathbf{b}-\mathbf{d}$ showing variation in metasomal colour pattern $\mathbf{b}$ voucher BF00928 from Vietnam c specimen from India d voucher BF00932 from Vietnam. 
Morphology. Head. Antenna with 54 flagellomeres.

Coloration. Bright ochreous yellow with flagellum, stemmaticum, metasomal tergite 4, hind coxa largely, hind femur medially, hind tibia apically, and hind tarsus black. In darker specimens black or dark brown marks occur on lateral lobes of mesoscutum, mesopleuron, propodeum antero-laterally, and metasomal tergites 2-4. Wings pale yellow with yellow to brown yellow venation and with fore wing parastigma and apex of $\mathrm{C}+\mathrm{SC}+\mathrm{R}$ black.

Notes. Differs from the three other sequenced Vietnamese specimens (A. spurivena sp. nov) for which CO1 sequences of more than 500 base pairs were obtained at 3 positions, each corresponding to a 3rd codon position (Table 1).

\section{Aleiodes spurivenatriplus Quicke \& Butcher, sp. n. urn:Isid:zoobank.org:act:766D5DBF-D67F-463A-81C8-F7F3DD334931 http://species-id.net/wiki/Aleiodes_spurivenatriplus}

Figure 1a

Holotype female. "MALAY PENIN: Selangor, Bukit Kutu, 3500 ft 11.9.1929, H. M. Pendlebury", "Ex F.M.S. Museum. B.M. 1955-354” (BMNH)

Coloration. Dark ochreous yellow [though coloration may have changed due to age of specimen] with black flagellum, stemmaticum, marks occur on lateral lobes of mesoscutum, mesopleuron, spot on metapleuron, metasomal tergite 2 except for triangular basal zone, tergites 3-4, hind leg except for trochanter and trochantellus. Wings pale yellow with yellow to brown yellow venation and with fore wing parastigma and apex of $\mathrm{C}+\mathrm{SC}+\mathrm{R}$ black.

Table I. Differentiating cytochrome oxidase 1 bases for two new species. Positions are numbered according to position in the Drosophila yakuba sequence (Clary and Wolstenholme 1985).

\begin{tabular}{llcc}
\hline & \multicolumn{3}{c}{ CO1 variable positions } \\
\cline { 2 - 4 } & $\mathbf{1 6 3 1}$ & $\mathbf{1 8 2 7}$ & $\mathbf{1 8 7 5}$ \\
\hline A. spurivena sp. n. (vouchers: BCLDQ00003, GenBank JF962592; & T & A & C \\
BF00928, GenBank JF903065; BF00932, GenBank JF90306) & C & G & T \\
\hline $\begin{array}{l}\text { A. spurivenaduplus sp. n. (voucher/holotype: BCLDQ01315; GenBank } \\
\text { HQ551252) }\end{array}$
\end{tabular}




\section{Acknowledgements}

We are very grateful to Kees van Achterberg (Naturalis Museum, Leiden) for the load of interesting Vietnamese material he collected. The Animal Systematic Research Unit and Integrated Ecology Lab, Department of Biology, Faculty of Science, Chulalongkorn University kindly allowed use of their Cell^ $\mathrm{D}^{\ominus}$ imaging facility. This work was partly supported by BRT (R152126) and a Chulalongkorn University Centenary Academic Development Project grant to BAB.

\section{References}

Areekul Butcher B, Quicke DLJ (2011) Revision of Aleiodes (Hemigyroneuron) parasitic wasps (Hymenoptera: Braconidae: Rogadinae) with reappraisal of subgeneric limits, descriptions of new species and phylogenetic analysis. Journal of Natural History.

Chen X, He J (1997) Revision of the subfamily Rogadinae (Hymenoptera: Braconidae) from China. Zoologische Verhandelingen 308:1-187.

Clary DO, Wolstenholme DR (1985)The mitochondrial DNA molecule of Drosophila yakuba: nucleotide sequence, gene organization, and genetic code. Journal of Molecular Evolution 22: 252-271. doi: 10.1007/BF02099755

Harris RA (1979) A glossary of surface sculpturing. State of California Occasional Papers in Entomology 28:1-31. 